\title{
Hipersexualidad asociada a pramipexol, en el tratamiento de síntomas parkinsonianos: revisión de la literatura, a propósito de 3 casos
}

\author{
Hypersexuality associated to pramipexole in the treatment of parkinsonian symptoms: \\ presentation of 3 cases and review of the literature
}

\author{
Nilton Custodio ${ }^{1 a, b, c}$, Rosa Montesinos ${ }^{1 a, d}$, David Lira ${ }^{1 a, c}$, Hernando Torres ${ }^{1 a, b}$ \\ ${ }^{1}$ Clínica Internacional. Lima. Perú.

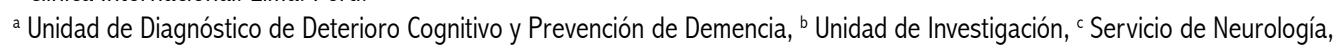 \\ ¿ Servicio de Medicina de Rehabilitación.
}

\section{Resumen}

Introducción: Los desórdenes del control de los impulsos han sido reportados en pacientes con enfermedad de Parkinson (EP), así como en usuarios de agonistas dopaminérgicos. Comunicamos tres casos de pacientes con síntomas parkinsonianos e hipersexualidad, un desorden del control de los impulsos, asociado al uso de pramipexol. Casos clínicos: Caso 1: Varón de 62 años de edad con EP en tratamiento con levodopa/carbidopa, que requirió inicio de pramipexol; a los doce meses presentó hipersexualidad que remitió parcialmente con escitalopram. Caso 2: Varón de 66 años de edad con EP en tratamiento con levodopa/carbidopa, entacapona y clonazepam; requirió inicio de pramipexol y a los dieciséis meses presentó hipersexualidad, que remitió totalmente con escitalopram. Caso 3: Varón de 45 años de edad con parkinsonismo secundario a encefalitis esclerosante aguda en tratamiento con risperidona, amitriptilina y clonazepam; requirió inicio de pramipexol; a los dos meses presentó hipersexualidad, recibió escitalopram, pero la hipersexualidad solo desapareció al disminuir la dosis de pramipexol; se inició levodopa/carbidopa y reapareció la hipersexualidad, que desapareció con la disminución de la dosis de levodopa/carbidopa. Conclusiones: Pramipexol podría estar asociado a hipersexualidad; pero, es necesario tener en cuenta las características relacionadas a cada enfermedad, así como los factores individuales. La hipersexualidad podría mejorar con el escitalopram, siendo necesario tratar los síntomas asociados.

Palabras clave: Agonistas dopaminérgicos; enfermedad de Parkinson; trastorno de control de impulsos; conducta sexual.

\begin{abstract}
Introduction: Impulse control disorders have been reported in patients with Parkinson's disease (PD), and in users of dopamine agonists. We report three cases of patients with parkinsonian symptoms and hypersexuality, an impulse control disorder associated with pramipexole use. Case reports: Case 1: A 62-year-old man with PD treated with levodopa/carbidopa. He needed to start pramipexole and twelve months later he presented hypersexuality that improved with escitalopram. Case 2: A 66-year-old man with PD treated with levodopa/carbidopa, entacapone and clonazepam. He needed to start pramipexole; sixteen months later he presented hypersexuality that disappeared with escitalopram. Case 3: A 45-year-old man with Parkinsonism secondary to acute disseminated encephalomyelitis treated with risperidone, amitriptyline and clonazepam. He needed to start pramipexole; two months later, he presented hypersexuality and received escitalopram; hypersexuality disappeared only when decreasing pramipexole dose; then he started levodopa/ carbidopa and hypersexuality reappeared that decreased again when reducing levodopa/carbidopa dose. Conclusions: Pramipexole may be associated with hypersexuality, but it is necessary to be careful with characteristics either related to disease or to individual factors. Hypersexuality may improve with escitalopram and its associated symptoms should be treated.
\end{abstract}

Key words: Dopamine agonists; Parkinson disease; impulse control disorders; sexual behavior.

\section{INTRODUCCIÓN}

Diversas conductas complejas han sido comunicadas en pacientes con enfermedad de Parkinson (EP), en las que se incluyen ludopatía, hipersexualidad y jugueteo, así como comprar, comer o usar medicación de manera compulsiva ${ }^{(1,2)}$. Estas conductas constituyen desórdenes del control de los impulsos (DCI), que son comportamientos compulsivos o repetitivos basados en la obtención de recompensa inmediata, desenfrenados y frecuentemente no planificados, que finalmente resultan en consecuencias negativas ${ }^{(3-5)}$. La prevalencia de los DCI en pacientes con EP varía de $4 \%$ a $8 \%$ $(1,4)$, y se duplica en pacientes usuarios de agonistas dopaminérgicos (AD) ${ }^{(1,5)}$; se observa DCI también en otras enfermedades, como el síndrome de piernas inquietas ${ }^{(6,7)} \mathrm{y}$ en usuarios de dosis altas de levodopa ${ }^{(8)}$.

La hipersexualidad, muchas veces ocultada por pacientes y familiares, debe cumplir los siguientes criterios ${ }^{(4)}$ : los pensamientos o conductas sexuales deben ser excesivos o existir un cambio atípico; puede existir preocupación bochornosa con los pensamientos sexuales, requerimiento sexual excesivo o inapropiado a la esposa o pareja sexual, promiscuidad inhabitual, masturbación compulsiva, requerimiento de líneas telefónicas sexuales, pornografía o pa- rafilias; la conducta tiene que persistir por más de un mes y las conductas deben originar alguna o más de las siguientes características: extrema preocupación, los intentos por controlar los pensamientos o las conductas fracasan o resultan en ansiedad, ocupan mucho tiempo, interfieren significativamente con el funcionamiento social u ocupacional y las conductas no deben ocurrir exclusivamente durante periodos de manía o hipomanía. La hipersexualidad en EP ha sido encontrada en $2,4 \%$ a $8,4 \%{ }^{(4,9)}$, pero las tasas varían dependiendo del evaluador y del método de evaluación empleado. La hipersexualidad ha sido descrita en pacientes con EP desde el advenimiento de la levodopa, en los 
años sesenta ${ }^{(10)}$, pero es más frecuente con el uso de $\mathrm{AD}^{(4,5,9)}$.

El pramipexol es un AD no ergótico, actúa selectivamente sobre los receptores $\mathrm{D}_{2}$, interacciona escasamente con receptores $\alpha$-adrenérgicos y serotoninérgicos. Dentro de la familia $\mathrm{D}_{2}$ tiene afinidad preferente por el receptor $\mathrm{D}_{3}{ }^{(11)}$. El pramipexol está indicado en el tratamiento de la EP idiopática y el síndrome de las piernas inquietas. La dosis de inicio recomendada es de 0,264 mg base al día, repartida en tres tomas, con incrementos progresivos según respuesta clínica y tolerancia del paciente, durante tres a cinco semanas, hasta una dosis máxima recomendada de 3,3 mg de base por día. Tiene una vida media plasmática de 8 a 12 horas y su vía de eliminación es renal. El pramipexol, al actuar selectivamente sobre receptores dopaminérgicos, presenta una menor proporción de efectos secundarios autonómicos, cardiovasculares y digestivos -como náuseas y vómitos, dispepsia, hipotensión ortostática, somnolencia y alucinaciones visuales-que otros AD ergóticos ${ }^{(12,13)}$. Los DCI, como la ludopatía y la hipersexualidad, han sido relacionados con el uso de AD en general, sin que se haya encontrado diferencias consistentes entre fármacos de este grupo, ni en su asociación con otros fármacos; tampoco se ha demostrado un efecto dosis dependiente. El pramipexol es un tratamiento seguro y eficaz en fases iniciales y avanzadas de la EP; permite retrasar la aparición de complicaciones motoras y es especialmente útil en la mejoría del temblor y la depresión; asimismo, muestra varios efectos de tipo neuroprotector en estudios de laboratorio ${ }^{(13,14)}$.

Comunicamos tres casos de pacientes con síntomas parkinsonianos e hipersexualidad asociada al uso de pramipexol, los que fueron evaluados en el servicio de neurología de la Clínica Internacional de Lima.

\section{CASOS CLÍNICOS}

\section{Caso 1}

Varón de 62 años de edad, jubilado, estuvo casado en una oportunidad y tuvo 4 convivientes; desde los 57 años de edad convive con una mujer 33 años menor.
Diagnosticado de EP a los 56 años de edad, recibía tratamiento con carbidopa/ levodopa 25/250 mg 4 veces al día. Debido a la progresión de síntomas motores, con un puntaje del unified Parkinson's disease rating scale (UPDRS) motor de 30 y fluctuaciones motoras, con predominio de fenómeno de fin de dosis. Se añadió pramipexol hasta $3 \mathrm{mg} /$ día, mejoraron los síntomas motores, llegando a un puntaje del UPDRS motor de 14. Doce meses después de iniciado el tratamiento con pramipexol, el paciente relató incremento del deseo sexual, empezó a comprar revistas y videos pornográficos, y asistía a prostíbulos dos o tres veces por semana. Iniciamos tratamiento con escitalopram 10 mg/día y la hipersexualidad disminuyó paulatinamente, sin desaparecer, al término de seis meses.

\section{Caso 2}

Varón de 66 años de edad, casado en una oportunidad. Diagnosticado de EP a los 56 años de edad, recibía tratamiento con carbidopa/levodopa 25/250 mg 5 veces al día, entacapona $200 \mathrm{mg}$ dos veces al día y clonazepam $2 \mathrm{mg} /$ día. El paciente presentaba un puntaje del UPDRS motor de 42 y fluctuaciones motoras, con predominio de discinesias. Fue hospitalizado para titulación lenta de pramipexol y se llegó a 4 mg/día al final del cuarto mes, obteniendo un puntaje del UPDRS motor de 20; las discinesias disminuyeron en frecuencia, pero no desaparecieron. Un año cuatro meses después de iniciado el tratamiento con pramipexol, la esposa refirió requerimiento sexual diario e intento de seducción a la cuidadora durante el aseo diario de genitales. Iniciamos tratamiento con escitalopram $10 \mathrm{mg} /$ día y la hipersexualidad disminuyó paulatinamente, hasta desaparecer al final del segundo mes.

\section{Caso 3}

Varón de 45 años de edad, casado en una oportunidad. Presentó a los 43 años de edad un cuadro clínico compatible con encefalomielitis diseminada aguda, tratado en un Instituto Neurológico de Argentina, siendo transferido a los dos meses a un centro de rehabilitación en el mismo país por un parkinsonismo secundario. Cuando fue evaluado en el servicio de neurología de la Clínica Internacional de Lima, el paciente presentaba facies hipomímica, dificultad para la articulación de las palabras y la deglución, marcada apatía, labilidad emocional y conductas motoras reiterativas; dificultad para incorporarse y mantenerse de pie, marcha dependiente, pasos cortos, descoordinados y asimétricos, hipertonía generalizada a predominio de miembros inferiores. Las imágenes de resonancia magnética cerebral mostraron focos de encefalomalacia en núcleo caudado y putamen bilateral y mitad derecha de protuberancia. Recibía tratamiento con risperidona $1 \mathrm{mg} /$ día, amitriptilina $25 \mathrm{mg} /$ día y clonazepam 0,5 mg/día. Se añadió al tratamiento pramipexol hasta $1 \mathrm{mg}$ tres veces al día y mejoraron los síntomas extrapiramidales. Dos meses después de iniciado el tratamiento con pramipexol, la esposa refirió que el paciente incrementó su requerimiento sexual y presentaba masturbación compulsiva. Iniciamos tratamiento con escitalopram $10 \mathrm{mg}$ dos veces al día, pero la hipersexualidad se incrementó, por lo que disminuimos progresivamente la dosis de pramipexol y continuamos con escitalopram, con lo cual desapareció la hipersexualidad, pero reaparecieron los síntomas extrapiramidales; por ello, iniciamos carbidopa/levodopa y mejoraron los síntomas motores, pero reapareció la hipersexualidad, la misma que disminuyó cuando reducimos la dosis de carbidopa/levodopa.

\section{DISCUSIÓN}

La asociación entre EP y DCI parece obedecer a una expresión clínica de la fisiopatología de EP, al resultado de las intervenciones usadas para tratar EP, o a una combinación de ambas. En esta serie corta de pacientes con síntomas parkinsonianos, existe una relación probable de hipersexualidad asociada al uso de pramipexol. Los dos primeros casos recibían levodopa previamente, mientras que en el tercer caso la hipersexualidad apareció solo con pramipexol y luego reaparecieron los mismos síntomas cuando se inició levodopa. Varias comunicaciones clínicas sugieren un rol importante de las terapias dopaminérgicas en la aparición de DCI en pacientes con EP (4,5,15,16), aunque existen algunos reportes que demuestran lo contrario ${ }^{(8)}$. 
El inicio del DCI puede ocurrir con el inicio o el incremento de la dosis de un $\mathrm{AD}$, y la remisión o reducción del $\mathrm{DCI}$, al reducir o cesar el $\mathrm{AD}^{(8,15,16)}$. Algunos estudios sugieren un rol importante en el desarrollo de DCI de dosis altas de levodopa ${ }^{(8)}$, inhibidores de monoamino oxidasa ${ }^{(17)}$, estimulación cerebral profunda ${ }^{(18)}$, y los AD como pramipexol ${ }^{(13)}$, ropinirol y pergolida ${ }^{(8,16)}$. La prevalencia de hipersexualidad asociada a monoterapia con levodopa es de $0,7 \%$ y con un $\mathrm{AD}$ puede llegar a $13,7 \%{ }^{(5,19)}$. En un estudio la hipersexualidad, fue más frecuente con pramipexol en relación a otros $\mathrm{AD}$, sin poder establecer una relación clara con la dosis de pramipexol ${ }^{(1)}$. En nuestra serie, 2 pacientes alcanzaron dosis de $3 \mathrm{mg} /$ día, mientras que un paciente llegó a los 4 $\mathrm{mg} /$ día. Respecto al tiempo de aparición de los síntomas y el inicio de pramipexol, observamos que, en el primer caso, los síntomas se iniciaron luego de 12 meses, en el segundo luego de 16 meses, y en el tercero luego de 2 meses. En un estudio de 15 pacientes varones la hipersexualidad empezó 8 meses después del inicio del $\mathrm{AD}^{(20)}$.

Una serie de casos ${ }^{(16)}$ y publicaciones (21) sugieren una particular asociación de DCI con pramipexol, un AD con especificidad para un receptor de dopamina D3, expresado en gran cantidad en las regiones límbicas ${ }^{(22)}$; sin embargo, estudios posteriores no han confirmado esta observación ${ }^{(5,9,19,23)}$. Los modelos animales de EP con exposición crónica a levodopa, en quienes se desarrolla conductas similares a compulsiones, no demuestran diferencia significativa con los expuestos a pergolida, ropinirol o pramipexol (24).

La ocurrencia de DCI, sugiere una susceptibilidad subyacente, la cual puede ser mediada por características relacionadas a EP o factores individuales. La ludopatía, la hipersexualidad y el uso compulsivo de medicación están asociados con inicio más joven de $\mathrm{EP}^{(23,25)}$. La diferencia de género también ha sido informada, siendo el sexo masculino más proclive a ludopatía e hipersexualidad; mientras que las mujeres tienen más probabilidad de desarrollar compras compulsivas ${ }^{(8,26)}$. En relación a factores sociales y culturales, se ha encontrado elevadas tasas de ludopatías en inmigrantes asiáticos (27). Los datos existentes sugieren que grupos específicos de individuos con EP son particularmente susceptibles a desarrollar algún DCI durante el curso de la enfermedad, particularmente durante el tratamiento con un AD. Los factores de riesgo posibles para el desarrollo de algún DCI en EP incluyen características clínicas relacionadas a compromiso premórbido del control de los impulsos, como una historia personal de algún DCI antes del inicio de $\mathrm{EP}^{(9)}$, historia personal o familiar de alcoholismo, fuertes rasgos de involucrarse en aventuras, hipomanía inducida por medicación e impulsividad no planificada ${ }^{(23)}$. En nuestro primer caso, consideramos que existieron rasgos premórbidos de hipersexualidad por el número de parejas sexuales y la diferencia de edad con su última pareja. En el tercer caso, se trata de un parkinsonismo secundario a un proceso infeccioso, con múltiples secuelas, que incluían trastornos conductuales y politerapia medicamentosa con risperidona, amitriptilina y clonazepam, que podrían ser considerados como factores de riesgo para el desarrollo de hipersexualidad, considerando que los síntomas también se presentaron con dosis baja de levodopa.

Ningún ensayo clínico ha sido realizado para identificar un medicamento seguro y eficaz para el tratamiento del DCI en EP. Recién en la última década se han realizado ensayos clínicos aleatorios de terapias para DCI, que han demostrado ser superiores a placebo e incluyen antagonistas de opioides e inhibidores de la recaptación de serotonina, como fluvoxamina y paroxetina ${ }^{(1,8)}$; sin embargo, los resultados no han sido consistentes. Basados en un ensayo abierto de escitalopram en el tratamiento de ludopatía (28), decidimos iniciar tratamiento con escitalopram $10 \mathrm{mg} /$ día en esta serie corta de pacientes con hipersexualidad asociada a pramipexol, con resultados favorables, pues los síntomas disminuyeron en el primer caso y desaparecieron en el segundo y tercer casos.

Respecto al manejo del DCI en EP, al inicio de la terapia de un $\mathrm{AD}$ se debería advertir a los pacientes sobre sus posibles efectos adversos e identificar los posibles factores de riesgo para su desarrollo, como: inicio en edad joven de EP, uso o abuso de alcohol e historia previa de desorden psiquiátrico. Posteriormente, cuando se identifica un DCI, se debería disminuir la dosis del AD, con el incremento progresivo de levodopa, tanto como sea necesario; si no disminuyen los síntomas, se podría realizar la rotación hacia otro $A D$ o retirar totalmente el AD. Además, se recomienda tratar las condiciones comórbidas, como ansiedad, depresión, psicosis, manía o demencia.

En conclusión, pramipexol podría estar asociado a hipersexualidad, pero es necesario tener en cuenta las características relacionadas a cada enfermedad, así como los factores individuales. La hipersexualidad podría responder favorablemente a escitalopram, siendo necesario hacer estudios que evalúen su eficacia y seguridad en el tratamiento de los DCI.

\section{REFERENCIAS BIBLIOGRÁFICAS}

1. Voon V, Potenza MN, Thomsen T. Medication related impulse control and repetitive behaviors in Parkinson's disease. Curr Opin Neurol. 2007;20:484-92.

2. Voon V, Fox SH. Medication-related impulse control and repetitive behaviors in Parkinson's disease. Arch Neurol. 2007;64:1089-96.

3. Klos KJ, Bower JH, Josephs KA, Matsumoto JY, Ahlskog JE. Pathological hypersexuality predominantly linked to adjuvant dopamine agonist therapy in Parkinson's disease and multiple system atrophy. Parkinsonism Relat Disord. 2005; 11:381-6.

4. Voon V, Hassan K, Zurowski M, de Souza M, Thomsen T, Fox $S$, et al. Prevalence of repetitive and reward-seeking behaviors in Parkinson's disease. Neurology. 2006;67:1254-7.

5. Voon V, Hassan K, Zurowski M, Duff-Canning S, de Souza M, Fox S, et al. Prospective prevalence of pathologic gambling and medication association in Parkinson's disease. Neurology. 2006;66:1750-2.

6. Driver-Dunckley ED, Noble BN, Hentz JG, Evidente VG, Caviness JN, Parish J, et al. Gambling and increased sexual desire with dopaminergic medications in restless legs syndrome. Clin Neuropharmacol. 2007;30:24955.

7. Tippmann-Peikert M, Park JG, Boeve BF, Shepard W, Silber MB. Pathologic gambling in patients with restless legs syndrome treated with dopaminergic agonists. Neurology. 2007;68:301-3.

8. Potenza MN, Voon V, Weintraub D. Drug insight: Impulse control disorders and dopamine therapies 
in Parkinson's disease. Nat Clin Pract Neurol. 2007;12:664-72.

9. Weintraub D, Siderowf AD, Potenza MN, Goveas J, Morales KH, Duda JE, et al. Association of dopamine agonist use with impulse control disorders in Parkinson's disease. Arch Neurol. 2006;63:969-73.

10.Stamey W, Jankovic J. Impulse control disorders and pathological gambling in patients with Parkinson's disease. The Neurologist. 2008;14:89-99.

11. Shannon KM, Bennett JP Jr, Friedman JH. Efficacy of pramipexole, a novel dopamine agonist, as monotherapy in mild to moderate Parkinson's disease. The Pramipexole Study Group. Neurology. 1997;49:724-8.

12. Moller JC, Oertel WH, Koster J, Pezzoli G, Provinciali L. Long-term efficacy and safety of pramipexole in advanced Parkinson's disease: results from a European multicenter trial. Mov Disord. 2005;20:602-10.

13. Izumi Y, Sawada H,Yamamoto N, Kume T, Katsuki $\mathrm{H}$, Shimohama $\mathrm{S}$, et al. Novel neuroprotective mechanisms of pramipexole, an anti-Parkinson drug, against endogenous dopamine-mediated excitotoxicity. Eur J Pharmacol. 2007;557:132-40.

14. Martínez-Corral M, Kulisevsky J. Pramipexol y enfermedad de Parkinson, una actualización. Rev Neurol. 2008;46:49-52.

15. Gschwandtner U, Aston J, Renaud S, Fuhr P. Pathological gambling in patients with Parkinson's Disease. Clin Neuropharmacol. 2001;24:170-2.

16. Dodd ML, Kloss KJ, Bower JH, Geda YE, Josephs KA, Ahlskog JE. Pathological gambling caused by drugs used to treat Parkinson disease. Arch Neurol. 2005;62:1377-81.
17. Galvez-Jimenez N. Rasagiline and selegiline induced hypersexuality and other impulse control disorders (ICD) in Parkinson's disease (PD). Presented at the Impulse Control Disorders in Parkinson's Disease Workshop: 2007 July 12-13, Toronto, ON, Canada.

18.Smeding HMM, Goudriaan AE, Foncke EMJ, Schuurman PR, Speelman JD, Schmand B. Pathological gambling after bilateral subthalamic nucleus stimulation in Parkinson's disease. J Neurol Neurosurg Psychiatry. 2007;78:517-9.

19. Lu C, Bharmal A, Suchowersky 0. Gambling and Parkinson's disease. Arch Neurol 2006;63:298.

20. Maia AF, Pinto AS, Barbosa ER, Menezes PR, Miguel EC. Obsessive-compulsive symptoms, obsessivecompulsive disorder, and related disorders in Parkinson's disease. J Neuropsychiatry Clin Neurosci. 2003;15:371-4.

21. Szarfman A, Doraiswamy PM, Tonning JM, Levine JG. Association between pathological gambling and parkinsonian therapy as detected in the Food and Drug Administration Adverse Event database. Arch Neurol. 2006;63:299-300.

22. Reuter J, Raedler T, Rose M, Hand I, Glascher J, Buchel C. Pathological gambling is linked to reduced activation of the mesolimbic reward system. Nat Neurosci. 2005;8:147-8.

23. Voon V, Thomsen T, Miyasaki JM, de Souza M, Shafro A, Fox SH, et al. Factors associated with dopaminergic medication-related pathological gambling in Parkinson's disease. Arch Neurol. 2007;64:212-6.

24. Fox SH, Visanji NP, Johnston TH, Gomez-Ramirez J, Voon V, Brotchie JM. Dopamine receptor agonists and levodopa and inducing psychosis-like behavior in the MPTP primate model of Parkinson disease. Arch Neurol. 2006;63:1343-4.

25. Evans AH, Lawrence AD, Potts J, Appel S, Lees AJ. Factors influencing susceptibility to compulsive dopaminergic drug use in Parkinson. Neurology. 2005;65:1570-4.

26. McElroy SL, Keck PE Jr, Pope HG Jr, Smith JM, Strakowski SM. Compulsive buying: a report of 20 cases. J Clin Psychiatry. 1994;55:242-8.

27. Petry NM. Gambling participation and problems among Southeast Asian refugees. Psychiatr Serv. 2003;54:1142-8.

28. Black DW, Shaw M, Forbush KT, Allen J. An openlabel Trial of escitalopram in the treatment of pathological gambling. Clin Neuropharmacol. 2007;30:206-12.

Manuscrito recibido el 4 de enero de 2010 y aceptado para publicación el 23 de febrero de 2010.
Correspondencia:
Dr. David Lira
Clínica Internacional
Av. Garcilazo de la Vega 1420
Lima 1, Perú.
Correo-e: davidlira@neuroconsultas.com 